\title{
Experience with Transcordal Silicone Stents in Adult Laryngotracheal Stenosis: A Bicentric Retrospective Study
}

\author{
Valerian Bourinet $^{\mathrm{a}} \quad$ Thibaut Raguin $^{\mathrm{b}} \quad$ Marc Fortin $^{\mathrm{a}} \quad$ Elsa Chetrit ${ }^{\mathrm{c}}$ \\ Julien Guinde ${ }^{a}$ Sophie Laroumagne ${ }^{a}$ Nicolas Fakhry ${ }^{d, e}$ Philippe Astoul ${ }^{a}$ e \\ Christian Debry ${ }^{b}$ Hervé Dutau ${ }^{a}$ \\ ${ }^{a}$ Department of Thoracic Oncology, Pleural Diseases, and Interventional Pulmonology, North Hospital of Marseille, \\ Aix-Marseille University, Marseille, France; ${ }^{b}$ Department of Otorhinolaryngology and Head and Neck Surgery, \\ University Hospital of Strasbourg, Strasbourg, France; ' Department of Radiology, North Hospital of Marseille, \\ Aix-Marseille University, Marseille, France; ${ }^{d}$ Department of Otorhinolaryngology and Head and Neck Surgery, \\ University Hospital of Marseille, Marseille, France; ${ }^{\mathrm{e}}$ Aix-Marseille University, Marseille, France
}

\section{Keywords}

Airway stenting $\cdot$ Benign laryngotracheal stenosis $\cdot$ Silicone stents - Therapeutic bronchoscopy

\begin{abstract}
Background: Benign stenosis involving laryngeal and upper tracheal structures represents a therapeutic challenge. Open surgery and endoscopic management have to be discussed by a multidisciplinary board in order to evaluate the risk and benefit for each patient. Objective: The objective of this retrospective study was to report the experience of two French centers with transcordal silicone stents (TSS) in the endoscopic management of benign laryngotracheal stenosis (BLTS) in adults, with focus on efficacy, safety, and tolerability. Methods: We performed a retrospective chart review of all cases of BLTS treated with TSS between January 2001 and June 2017 at two tertiary centers in France: the Centre Hospitalier Régional Universitaire de Strasbourg and the Hôpital Nord de Marseille. Results: A total of 17 patients were included. Eleven had a tracheostomy at initial management which consisted of 8 T-tubes and 9 strictly endoluminal stents placements. The main complications were minor aspirations in 5 patients (29\%), granulation in 3 patients (18\%),
\end{abstract}

migration in 2 patients (12\%), and severe dysphonia in 3 patients (18\%). After a mean duration of 18.3 months, 11 patients (65\%) had had their TSS definitely removed, 13 patients were tracheostomy free (76\%), and a TSS remained in place in 4 patients (24\%). Conclusions: Adult BLTS treatment with TSS placement is associated with low morbidity and excellent clinical outcomes, with a large proportion of patients free of airway instrumentation on long-term follow-up.

(c) 2018 S. Karger AG, Basel

\section{Introduction}

The most frequent etiologies of benign tracheal stenosis (BTS) include endotracheal intubation, tracheostomy, tracheal surgery, radiation therapy, caustic inhalation, tracheal trauma, and idiopathic [1].

The gold standard treatment for symptomatic BTS if the thyroid cartilage is not involved is tracheal sleeve resection. Success rates in the $70-94 \%$ range have been reported, with perioperative mortality rates in the 1.5-9\% range [2-7]. Sufficient residual tracheal tissue must remain to minimize the tension on the anastomosis and to prevent anastomotic complications.

\section{KARGER}

(c) 2018 S. Karger AG, Basel

E-Mail karger@karger.com

www.karger.com/res
Hervé Dutau, MD

Department of Thoracic Oncology, Pleural Diseases, and Interventional Pulmonology North Hospital of Marseille, chemin des Bourrely

FR-13915 Marseille Cedex 20 (France)

E-Mail hdutau@ap-hm.fr 
Fig. 1. Stents used. a Straight silicone stent. b Hourglass-shaped silicone stent. c Montgomery T-tube.
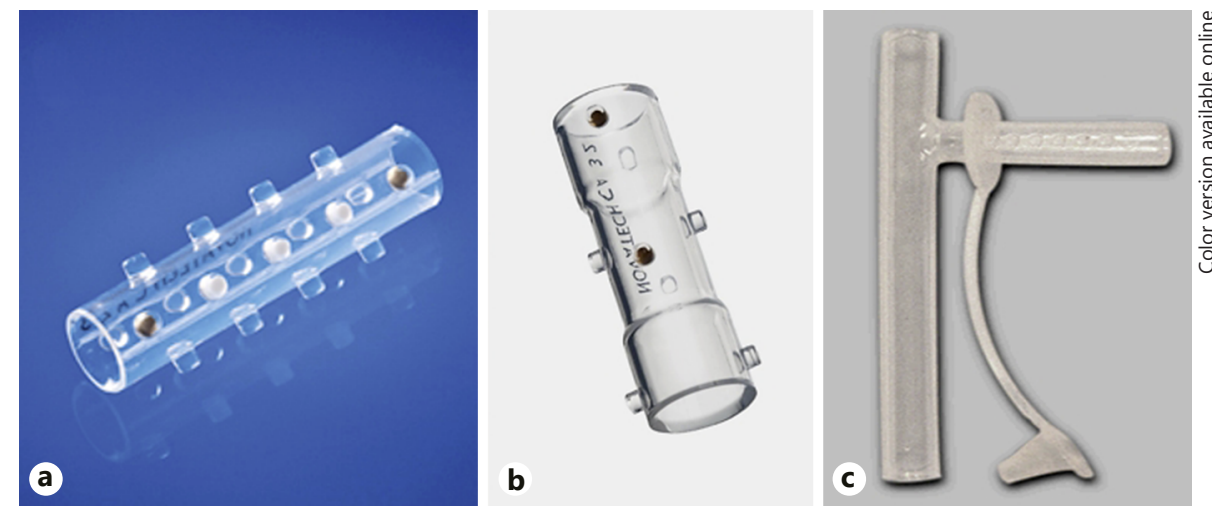

Although surgery is the gold standard treatment for most BTS, endoscopic treatment (ET) also plays a central role in the treatment of BTS. ET should be performed first in patients who present with respiratory distress or in patients with severe stenosis who will undergo surgery to facilitate endotracheal intubation and relieve symptoms. ET should also be considered as a first-line treatment in simple BTS, i.e., without cartilaginous involvement, as a great proportion of patients with simple BTS will require no further therapy after one ET session $[8,9]$. The success rates in complex BTS, i.e., with cartilaginous involvement, are lower in the $70 \%$ range, but remain interesting for patients who are not surgical candidates because of comorbidities or technical anatomic reasons [8, 10-14].

ET involves mechanical dilatation of the stenosis by rigid bronchoscope or balloon, which can be followed if necessary by silicone or self-expandable fully covered metal stent placement, depending on the risk of restenosis [15]. The main shortcoming of stents in BTS is the high risk of migration $[10,12,14]$, a risk which is particularly important when the stenosis is located close to the vocal cords. Our experience, which has been shared by many, has shown that it is imperative to leave at least $1 \mathrm{~cm}$ between the upper margin of a stent and the vocal cords to prevent discomfort and obstructive complications such as laryngeal edema, stent migration, and granulation tissue formation just below the vocal cords, which could be fatal. This makes high BTS, within $2 \mathrm{~cm}$ of the vocal cords, a subgroup in which ET has traditionally had poor success due to the impossibility to stent. Many patients in this group have been treated with definitive tracheostomies to bypass the stenotic area. Surgery can be considered, but is more complex. A preoperative tracheostomy is generally required in patients not previously tracheotomized. Even though morbidity and mortality are considered low, surgery can complicated by stenosis recurrence, dehiscence, and death [16]. Transcordal stents represent an alternative to tracheostomy in this population when simple dilatation is insufficient. Transcordal stenting has previously been described mainly in patients with a tracheostomy complicated by recurrent aspirations as well as in the postoperative course of laryngotracheal surgeries, such as temporary splinting [1719]. Very few patients with benign laryngotracheal stenosis (BLTS) have previously been treated with transcordal stenting [17-19].

The objective of this retrospective study was to report the experience of two French centers with transcordal silicone stents (TSS) in the management of BLTS with focus on efficacy, safety, and tolerability.

\section{Patients and Methods}

We performed a retrospective chart review of all cases of TSS performed at two tertiary centers in France: the Centre Hospitalier Régional Universitaire de Strasbourg and the Hôpital Nord de Marseille.

All cases with TSS procedures performed between January 2001 and June 2017 with straight (TD) and hourglass-shaped (HG) Dumon Silicone stents (Novatech GSS TD and ST ${ }^{\circledR}$; Novatech SA, La Ciotat, France) as well as Montgomery T-tubes (Montgomery Safe-T-Tube ${ }^{\circledR}$; Boston Medical Products, Shrewsbury, MA, USA) (Fig. 1) were included.

Malignant stenoses were excluded as well as cases where the TSS were inserted for recurrent aspirations and in whom the proximal end of the stent was below the vocal cords.

Charts were reviewed for stenosis characteristics (length, location, degree of obstruction), stent characteristics (type, dimensions), stent tolerability (pain, cough), and stent-related complications (dysphagia, granuloma formation, mucus plugging, migration) as well as patient evolution, including the possibility of definitive stent removal. 
Fig. 2. Proximal end of a transcordal silicone stent seen at the level of the arytenoid cartilages endoscopically (a) and on imaging (b).
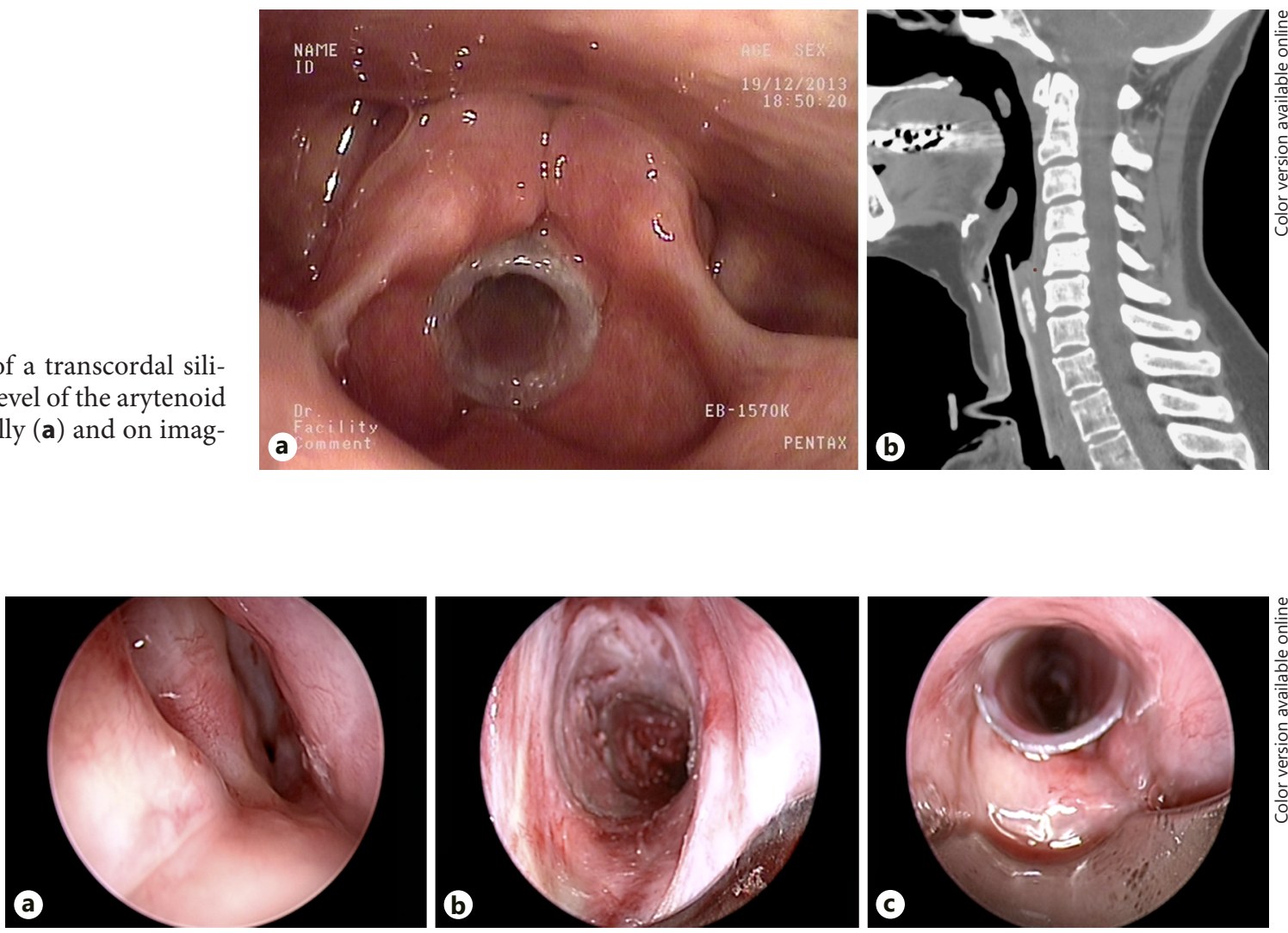

Fig. 3. Postradiation tracheal stenosis. a Initial aspect. b After mechanical dilatation. c After transcordal silicone stent placement.

The insertion technique differed between the two centers. At the Centre Hospitalier Régional Universitaire de Strasbourg, stents were inserted during suspension laryngoscopy, using a foreign body forceps. TD and HG stents were used. The operator aimed to place the middle of the stent at the level of the cricoid cartilage and the proximal end at least at the level of the arytenoid cartilages or slightly higher if possible (Fig. 2). A safety wire was attached to the stent in case the stent needed to be removed urgently, and a temporary tracheostomy was used if stent positioning was difficult to allow ventilation during the procedure. At the Hôpital Nord de Marseille, the technique used differed. Rigid bronchoscopy was performed to mechanically dilate the stenosis with progressively larger rigid bronchoscopes (Fig. 3, 4). If patients had a tracheostomy in place, a Montgomery T-tube was used while a straight silicone stent was used in patients without tracheostomy. In patients with a tracheostomy in place, if the Montgomery T-tube was well tolerated for 2-3 months, it was replaced by a straight silicone stent. In our practice, HG stents were chosen in case of short stenosis (no longer than $2 \mathrm{~cm}$, which is the length of the central part of these stents). For longer stenosis, a TD stent was chosen. Both centers used nebulized normal saline three times daily after stent placement as long as the stent was left in place.

The follow-up schedule for clinical evaluations and bronchoscopy was tailored to each patient's need and left to the discretion of the clinician.

Transcordal Silicone Stents in Adult Laryngotracheal Stenosis
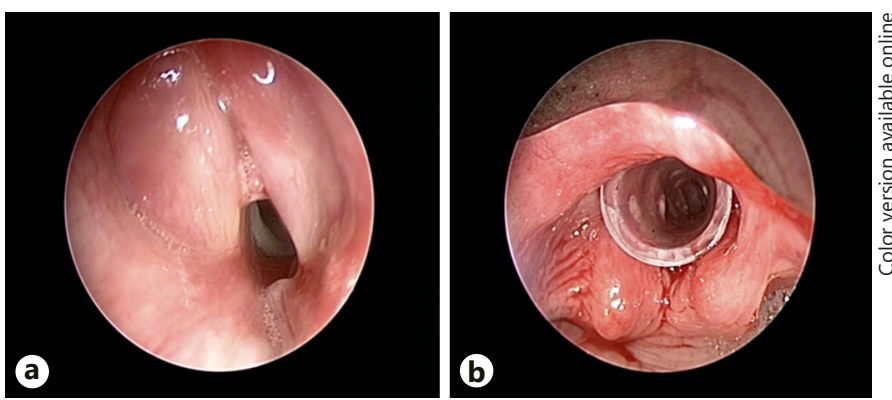

Fig. 4. Posttraumatic benign tracheal stenosis before (a) and after transcordal silicone stent placement (b).

\section{Results}

From January 2001 to June 2017, 17 TSS were inserted at the Hôpital Nord de Marseille and the Centre Hospitalier Régional Universitaire de Strasbourg. The most frequent stenosis etiologies were tracheostomy and endotracheal intubation in 6 patients (35\%), post tracheal surgery 
Table 1. Demographic data, etiologies, endoscopic management, and follow-up

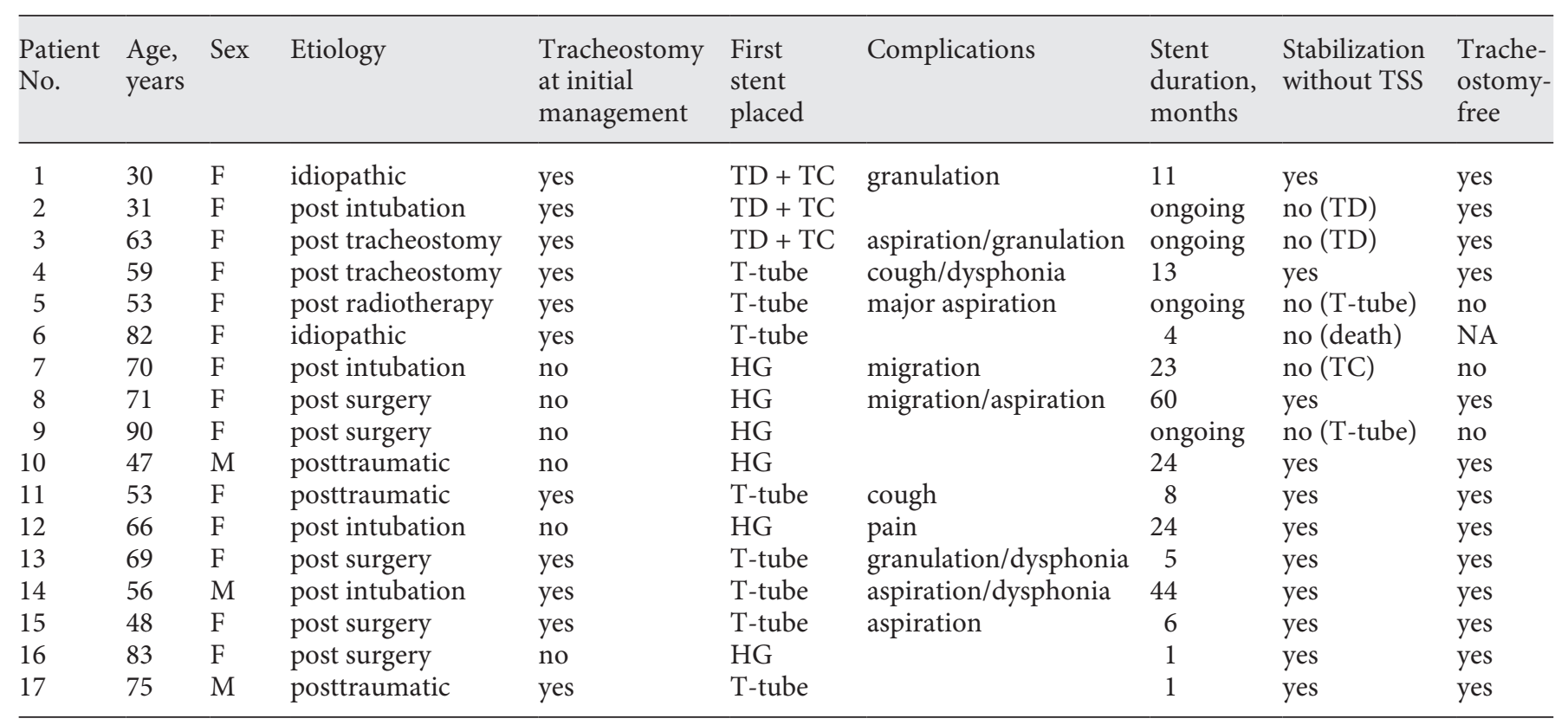

HG, hourglass-shaped stent; NA, not applicable; TC, tracheostomy canula; TD, tracheal Dumon stent; TSS, transcordal silicone stent.

in $5(29 \%)$, trauma in $3(18 \%)$, idiopathic in $2(12 \%)$, and post radiation in 1 (Table 1 ). All stenoses were complex. Six patients did not have tracheostomies at baseline and were dyspneic at rest, while the other 11 had tracheostomies in place at their initial endoscopic procedure and were asymptomatic.

The 6 patients without tracheostomy at baseline were treated with HG stents. At initial ET, 3 patients had 14-, $12-$, and 14-mm stents placed while the other 3 had 12-, $10-$, and $12-\mathrm{mm}$ stents placed. Amongst the 11 patients with a tracheostomy at baseline, 8 were initially treated with a T-tube (diameters $11-13 \mathrm{~mm}$ ) while the other 3 were treated with TD stents (diameters 10 and $12 \mathrm{~mm}$ ) which were positioned above the tracheostomy that was left in place to ensure maximal patient security.

Minor complications were frequent. Aspiration was noted by 5 patients (29\%) but was minor in all but 1 patient $(6 \%)$ in whom it led to a change in stent diameter. Three patients (18\%) had granulation tissue formation at the end of their stent which required nonurgent ET. Two (12\%) migration incidents occurred (2 HG stents), one of which was corrected by placing a larger stent while the other was corrected by replacing a T-tube. Dysphonia was present in all patients, who could speak at the sound intensity of a whisper except for 3 patients (18\%) who could not produce a sufficient sound intensity to be understood when speaking. Significant cough was present in 2 patients (12\%). One patient (6\%) had laryngeal discomfort which resolved with stent removal.

After a mean duration of 18.3 months, 11 patients (65\%) had had their TSS removed without necessity to reinsert it after a follow-up of at least 1 year. Four patients (24\%) remained with their stent in place, 3 of whom had had a stent removal attempt resulting in restenosis after having their stent in place for 35, 36, and 113 months, respectively. One patient $(6 \%)$ has had his stent in place for 12 months and no removal has been attempted. One patient (6\%) died 4 months after his TSS had been placed from a cause unrelated to his airway disease.

Amongst the 11 patients with a tracheostomy at baseline, 1 (9\%) died of unrelated reasons and 1 (9\%) still has a T-tube. The remaining 9 patients (73\%) all had their tracheostomy orifice closed and only 1 patient remains with a silicone stent. Amongst the 6 patients without tracheostomy at baseline, 2 eventually required a tracheostomy to be performed because of TSS failure. One patient now has a T-tube while the other has a definitive tracheostomy. The other 4 patients remain tracheostomy and TSS free. 


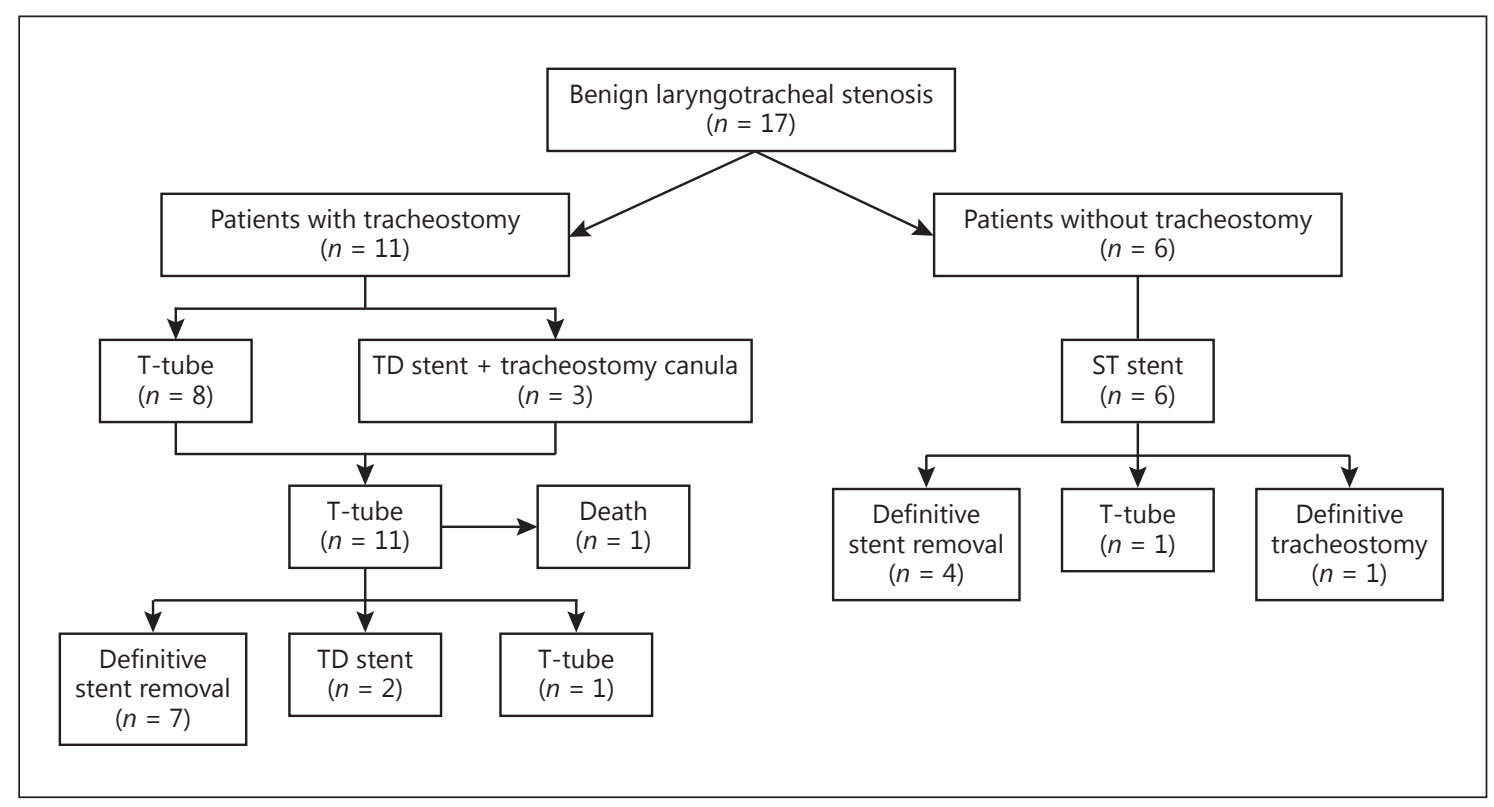

Fig. 5. Patient flowchart.

Of the total group, 13 patients $(76 \%)$ are now tracheostomy free, 2 have a T-tube, and 1 has a definitive tracheostomy. Figure 5 summarizes the evolution of all patients.

\section{Discussion}

Adult BTS is a complex entity with multiple etiologies [1]. For pure BTS, surgical management remains the gold standard [20,21], but the risks and benefits should always be evaluated by a multidisciplinary board, and in certain situations the risk-benefit evaluation may favor an endoscopic approach.

In BLTS, there is a lack of recommendations, given the small size of surgical and endoscopic series already published and the heterogeneity of anatomic lesions [22].

The drawback of the surgical approach is its risk of major complications, such as anastomotic dehiscence, in patients with benign disease and generally prolonged survival. The complexity of surgical procedures and the risk of complications increases with proximity to the vocal cords and with involvement of the cricoid and thyroid cartilage.

Historically, patients with BLTS with insufficient subglottic space for stenting and failure of simple endoscopic dilatation who were not candidates for surgical treatment had no other alternative treatment options than de- finitive tracheostomy. We present here our experience with an alternative treatment option: transcordal silicone stenting. This approach is not associated with the esthetic and psychological impacts of a tracheostomy orifice, unless a T-tube is used, and allows airway instrumentation weaning in a significant proportion of patients.

Tracheostomy certainly remains the gold standard [23] in emergency situations and in certain situations as a preventive safety measure, such as in ENT radiation therapy, but an alternative exists for certain patients.

Total laryngectomy is now seldom performed because of its ravaging consequences, except in palliative situations where definitive tracheostomies for supraglottic lesions cause repeated aspiration episodes.

When a surgical approach is chosen for BTS, tracheotracheal resection anastomosis or cricotracheal resection with thyrotracheal anastomosis are the treatments of choice for grade 3 and 4 stenosis [24] of the Myer-Cotton classification [25]. If the stenosis is purely tracheal, tracheotracheal resection anastomosis is the procedure of choice [20]. Cricotracheal resection [20] remains the most frequently performed surgery in patients with laryngotracheal stenosis without vocal cord involvement. First the trachea is exposed to allow the evaluation of the cricoid cartilage. If it is not involved in the stenosis, a cricotracheal anastomosis will be performed, but if the cartilage is involved in the stenosis, the procedure performed will vary in order to keep the largest part of the cartilage. 
If none of the cricoid cartilage is preservable, a thyroarytenotracheal anastomosis can be performed, with sutures anchoring the arytenoids to the posterior face of the trachea. Subtotal cricoid resections cause laryngeal immobility because of muscle insertion removal. Hence, a postoperative tracheotomy is required with a second procedure to regain vocal cord mobility.

Cricoid enlargement procedures have also been described [26-28] and allow a surgical approach when the glottic space is involved. They consist of the insertion of a piece of cartilage in a vertical full-height incision of the posterior cricoid, which causes posterior enlargement of the cricoid space. If required, a similar procedure can be performed anteriorly.

Posterior cordotomy and cordectomy without other interventions should not be used to treat laryngotracheal stenosis. Their effect is limited to the glottic space, without impact on the supra- or subglottic space. Cordectomies can also be complicated by anterior vocal cord synechia, which further reduces the glottic lumen [29].

All surgical procedures require a meticulous preoperative evaluation and a thorough discussion of morbidity and mortality with the patient. The risks of stenosis recurrence, anastomosis dehiscence, and mediastinal infections should be discussed $[20,30]$.

In cases where surgical risk outweighs benefit, ET can offer symptom palliation and may even be curative. For simple BTS, the results of ET with mechanical dilatation are well documented. In simple stenosis, i.e., without cartilaginous involvement, the success rate approaches $100 \%$ $[8,9]$. For complex stenosis, i.e., with cartilaginous involvement, ET with mechanical dilatation followed by nondefinitive stent insertion (fully covered metallic or silicone) has also been reported to give interesting results. Without stenting, restenosis rates are excessively high. Mitomycin C [31] and corticosteroid [32] application have not proved to prevent restenosis.

Removable stent placement for a mean duration of 18 months demonstrated a $70 \%$ success rate $[8,9]$, but requires long-term management with nebulizations of saline and exposes the patients to stent-related complications. Stent migration is the most frequent complication. Alternative methods of external fixation of the stent in high BTS are an option if this problem occurs [33-37]. Given that our patients had a pathological area between the vocal cords and the cricoid, this option could not be considered.

For patients presenting with BLTS, endoscopic options rely on repeated dilatations, possibly assisted by laser [14]. Stent placement would impose covering of the glottic area, with the proximal edge of the stent above the vocal cords and below the epiglottis. One could fear bad clinical tolerance with laryngeal discomfort and repeated aspirations.

We were reassured by Monnier's work from 2003 [17], which described the feasibility and tolerability of the use of a laryngotracheal silicone stent, the Easy-LT-Mold. This stent was used for postsurgical calibration in laryngotracheal stenosis in 10 patients. Tolerance was excellent and no aspirations were reported, but stents were only left in place for a limited period of time ranging from 1 week to 3 months. This stent is not commercially available. Chambres et al. [18] also reported the use of HG TSS in 10 patients, 5 of whom had laryngotracheal stenosis. Stents were left in place for a mean duration of 6 months and no significant complications were reported. Finally, Alshammari and Monnier [19] published a prospective cohort of 65 patients, 53 of whom were of pediatric age, with BLTS who were treated with Easy-LT-Mold stents. Stents were used for postsurgical airway calibration again, but also after pure ET. Tolerance was again excellent and stents were removed after a median follow-up of 23 months in $83 \%$ of patients, without the need for re-intervention during follow-up.

Our study is the first to focus solely on benign adult stenosis. Different stent types were used when compared to previous studies, in part because Easy-LT-Mold stents are not commercially available, but also because of the previous experience of the two participating centers with Dumon-type silicone stents. It is also important to note that the two participating centers and services are from different specialties, pulmonary medicine and otorhinolaryngology, which explains the different insertion techniques and stents used. In Marseille, rigid bronchoscopy and TD stents were used, while in Strasbourg, HG stents and suspension laryngoscopy were used. Also, due to the different training backgrounds, the group in Marseille did not initially feel secure removing the tracheostomy and installing a straight silicone stent, hence for their first 3 cases, the tracheostomy was initially left in place. For their 3 subsequent cases, the tracheostomy was removed at the initial procedure.

When comparing the complication profile with that of traditional tracheal stents which do not protrude through the vocal cords, we rapidly noticed that the migration rate was lower in our study, which may be easily explained by the frequent use of T-tubes. On the other hand, aspirations and dysphonia are complications not encountered with traditional airway stents. Aspirations were frequent (29\%) but caused a change in therapeutic approach in 
only 1 patient (6\%). This low rate of clinically significant aspirations is explained by the technique of stent positioning. The proximal end of the stent is precisely positioned slightly higher than the arytenoid cartilages, but not too high above them, to allow normal epiglottic movement and proper sealing of the airway when swallowing. Dysphonia is the main drawback of TSS and was present in all patients, but severe in only 3 patients (18\%). It easily resolves with stent removal and the help of a speech therapist. It is also interesting to note that longterm instrumentation-free airway rates are similar with TSS and traditional airway stents and that $75 \%$ of patients had their previous "permanent" tracheostomy removed.

The main limitations of this study include its retrospective nature, the small number of patients included, as well as the heterogeneity of stents and insertion techniques used.

In conclusion, high BTS treatment with TSS placement is associated with low morbidity and excellent clinical outcomes, with a large proportion of patients free of airway instrumentation on long-term follow-up. Hence, TSS represent an interesting alternative to permanent tracheostomy and surgery when complex surgical proce- dures with higher morbidity are required. All cases of BTS should be discussed with endoscopists and surgeons experienced in the treatment of this pathology. Treatment should be tailored according to the patient's fitness to undergo surgery and to the complexity of the required surgical procedure. More prospective studies are required to define the ideal stent and appropriates indications for TSS. With the anatomic complexity of the laryngotracheal region, 3D-printed customized stents certainly represent an interesting avenue to explore.

\section{Statement of Ethics}

This study was approved by the institutional review boards of the Centre Hospitalier Régional Universitaire de Strasbourg and the Hôpital Nord de Marseille.

\section{Financial Disclosure and Conflicts of Interest}

H. Dutau is consultant for Novatech SA. All the remaining authors have no conflict of interest to disclose related to this publication.

\section{References}

1 Lorenz RR: Adult laryngotracheal stenosis: etiology and surgical management. Curr Opin Otolaryngol Head Neck Surg 2003;11:467-472.

2 Anand VK, Alemar G, Warren ET: Surgical considerations in tracheal stenosis. Laryngoscope 1992;102:237-243.

3 Grillo HC, Donahue DM, Mathisen DJ, et al: Postintubation tracheal stenosis. Treatment and results. J Thorac Cardiovasc Surg 1995; 109:486-492.

4 París F, Borro JM, Tarrazona V, et al: Management of non-tumoral tracheal stenosis in 112 patients. Eur J Cardiothorac Surg 1990;4 265-268; discussion 268-269.

5 Maggi G, Ardissone F, Cavallo A, et al: Tracheal stenosis. A study of 100 cases. Int Surg 1990;75:225-230.

6 6 Bonnette P, Colchen A, Leroy M, et al: Tracheal resection-anastomosis for iatrogenic stenosis. Experience in 340 cases. Rev Mal Respir 1998;15:627-632.

7 Rea F, Callegaro D, Loy M, et al: Benign tracheal and laryngotracheal stenosis: surgical treatment and results. Eur J Cardiothorac Surg 2002;22:352-356.

8 Dalar L, Karasulu L, Abul Y, et al: Bronchoscopic treatment in the management of benign tracheal stenosis: choices for simple and complex tracheal stenosis. Ann Thorac Surg 2016;10:1310-1317.
-9 Galluccio G, Lucantoni G, Battistoni P, et al: Interventional endoscopy in the management of benign tracheal stenoses: definitive treatment at long-term follow-up. Eur J Cardiothorac Surg 2009;35:429-433.

10 Brichet A, Verkindre C, Dupont J, et al: Multidisciplinary approach to management of postintubation tracheal stenoses. Eur Respir J 1999;13:888-893.

11 Cavaliere S, Bezzi M, Toninelli C, et al: Management of post-intubation tracheal stenoses using the endoscopic approach. Monaldi Arch Chest Dis 2007;67:73-80.

12 Martinez-Ballarin JI, Diaz-Jimenez JP, Castro MJ, et al: Silicone stents in the management of benign tracheobronchial stenoses. Tolerance and early results in 63 patients. Chest 1996; 109:626-629.

13 Maldonado F, Loiselle A, Depew ZS, et al: Idiopathic subglottic stenosis: an evolving therapeutic algorithm. Laryngoscope 2014;124: 498-503.

14 Bolliger CT, Probst R, Tschopp K, et al: Silicone stents in the management of inoperable tracheobronchial stenosis: indications and limitations. Chest 1993;104:1653-1659.

15 Fortin M, Lacasse Y, Elharrar X, et al: Safety and efficacy of a fully covered self-expandable metallic stent in benign airway stenosis. Respiration 2017;93:430-435.
16 D'Andrilli A, Venuta F, Rendina EA: Subglottic tracheal stenosis. J Thorac Dis 2016;8 (suppl 2):S140-S147.

17 Monnier P: A new stent for the management of adult and pediatric laryngotracheal stenosis. Laryngoscope 2003;113:1418-1422.

18 Chambres O, Schultz P, Debry C: The Larynxane ST intralaryngeal endoprosthesis for laryngotracheal pathologies. J Laryngol Otol 2006;120:942-948.

19 Alshammari J, Monnier P: Airway stenting with the LT-Mold ${ }^{\mathrm{TM}}$ for severe glotto-subglottic stenosis or intractable aspiration: experience in 65 cases. Eur Arch Otorhinolaryngol 2012;269:2531-2538.

20 George M, Lang F, Pasche P, et al: Surgical management of laryngotracheal stenosis in adults. Eur Arch Otorhinolaryngol 2005;262: 609-615.

21 Rubikas R, Matukaitytè I, Jelisiejevas JJ, et al: Surgical treatment of non-malignant laryngotracheal stenosis. Eur Arch Otorhinolaryngol 2014;271:2481-2487.

22 Lewis S, Earley M, Rosenfeld R, et al: Systematic review for surgical treatment of adult and adolescent laryngotracheal stenosis. Laryngoscope 2017;127:191-198.
Transcordal Silicone Stents in Adult Laryngotracheal Stenosis 
23 Gadkaree SK, Pandian V, Best S, et al: Laryngotracheal stenosis: risk factors for tracheostomy dependence and dilation interval. Otolaryngol Head Neck Surg 2017;156:321-328.

24 Sandu K, Monnier P: Cricotracheal resection. Otolaryngol Clin North Am 2008;41:981998.

25 Myer CM, O’Connor DM, Cotton RT: Proposed grading system for subglottic stenosis based on endotracheal tube sizes. Ann Otol Rhinol Laryngol 1994;103:319-323.

26 Pradhan T, Kapil S, Thakar A: Posterior cricoid split with costal cartilage augmentation for high subglottic stenosis. Indian J Otolaryngol Head Neck Surg 2008;60:147-151.

27 Keghian J, Lawson G, Orban D, et al: Composite hyoid-sternohyoid interposition graft in the surgical treatment of laryngotracheal stenosis. Eur Arch Otorhinolaryngol 2000; 257:542-547.
28 Baujat B, Struk S, Lesnik M, et al: Fascia temporalis free flap for cricotracheal reconstruction: a novel approach. Ann Thorac Surg 2017;104:1040-1046.

29 Naunheim MR, Song PC, Franco RA, et al: Surgical management of bilateral vocal fold paralysis: a cost-effectiveness comparison of two treatments. Laryngoscope 2017;127:691697.

30 Ciccone AM, De Giacomo T, Venuta F, et al: Operative and non-operative treatment of benign subglottic laryngotracheal stenosis. Eur J Cardiothorac Surg 2004;26:818-822.

31 Eliashar R, Gross M, Maly B, et al: Mitomycin does not prevent laryngotracheal repeat stenosis after endoscopic dilation surgery: an animal study. Laryngoscope 2004;114:743746.

32 Perepelitsyn I, Shapshay SM: Endoscopic treatment of laryngeal and tracheal stenosis - has mitomycin $\mathrm{C}$ improved the outcome? Otolaryngol Head Neck Surg 2004; 131:16-20.
33 Colt HG, Harrell J, Neuman TR, et al: External fixation of subglottic tracheal stents. Chest 1994;105:1653-1657.

34 Miwa K, Takamori S, Hayashi A, et al: Fixation of silicone stents in the subglottic trachea: preventing stent migration using a fixation apparatus. Ann Thorac Surg 2004;78:21882190.

35 Majid A, Fernandez-Bussy S, Kent M, et al: External fixation of proximal tracheal airway stents: a modified technique. Ann Thorac Surg 2012;93:e167-e169.

36 Musani AI, Jensen K, Mitchell JD, et al: Novel use of a percutaneous endoscopic gastrostomy tube fastener for securing silicone tracheal stents in patients with benign proximal airway obstruction. J Bronchology Interv Pulmonol 2012;19:121-125.

37 Dhooria S, Agarwal R: External fixation of a subglottic tracheal silicone stent. Ann Am Thorac Soc 2014;11:467-468. 